\title{
LA COMPIUTA DONZELLA: UNA VOZ FEMENINA EN EL TRECENTO ITALIANO
}

\author{
LA COMPIUTA DONZELLA: A FEMINIST VOICE IN THE ITALIAN TRECENTO
}

Ana $\mathrm{M}^{\mathrm{a}}$ Domínguez Ferro

Universidad de Santiago de Compostela

\section{Resumen:}

El objeto del presente trabajo es, en primer lugar, presentar dos sonetos de dudosa atribución a una "Compiuta Donzella di Firenze", pertenecientes al corpus de la poesía cortés toscana y septentrional, en los que una voz femenina se muestra contraria a los deseos del padre. Además, pretendemos analizar las conexiones de estos textos con otros códigos líricos románicos, como la canción de mujer o las poesías de las trobairitz.

\section{Palabras claves:}

"Compiuta Doncella di Firenze", Trecento, Italy, sonetos.

\section{Abstract:}

This paper intends, first, to study two sonnets, allegedly written by a certain "Compiuta Doncella di Firenze". These sonnets belong to the corpus of Toscan and Northern courtly love poetry and in them a female voice opposes her father's desires. We will also analyse the connections between these texts and other Romanesque Lyric conventions such as the woman's songs or the trobairitz's poems/lyrics.

\section{KEY WORD:}

"Compiuta Doncella di Firenze", Trecento, Italy, sonnets. 


\section{INTRODUCCIÓN}

Bajo el nombre de la Compiuta Donzella di Firenze se conservan tres composiciones poéticas en el cancionero Vaticano lat. 3793 que, supuestamente, designaría a una poetisa del siglo XIII de la que no conservamos noticias. Precisamente por ello, es también difícil precisar si la rúbrica hace referencia a un nombre auténtico, a un pseudónimo, o a un senhal, dado que el adjetivo 'compiuta' es un término utilizado en las alabanzas morales de la dama, dentro de la lírica amorosa, para hacer referencia a una dama dotada de todas las cualidades ${ }^{2}$. Pero además de la ambigua referencia con la que se rubrican las composiciones, otros textos recogidos en el mismo cancionero parecen aludir a ella, como los sonetos de Maestro Torrigiano Esser donzella di trovare dotta (PST; 45.4) y S'una donzella di trovar s'ingegna (PST; 45.5) o las composiciones que forman parte de la tenzone entre la Compiuta donzella con otro autor: Gentil donzella somma ed insegnata y Perc'ogni gioia chè rara è graziosa (PDI, p. 436-437)³. Otra canción en la órbita de las anteriores es una composición de dudosa atribución, Gentil donzella, di pregio nomata ${ }^{4}$. El texto, que desarrolla el tópico de la alabanza a la dama, contiene en el exordio una referencia que parece remitir claramente a nuestra protagonista: né si compiuta de tutto valore(PDII, 474, v. 4).

Además de estas alusiones en el corpus poético, Guittone D'Arezzo dirige una carta en prosa a una "soprapiacente donna, di tutto compiuto savere, di pregio coronata, degna mia Donna Compiuta, Guitton, vero devotissimo fedel vostro, de quanto el vale e pò, umilemente se medesmo racomanda voi". (Margueron 1990: 87)

Tras la salutatio de la misiva, frate Guittone recomienda a la Compiuta el servicio a Dios: “Or dunque, gentile mia Donna, quanto el Signor nostro v'ha maggiormente allumata e smirata a compimento de tutta preziosa vertute più ch'altra donna terrena,

1 Este trabajo es una versión ampliada del estudio "Una voz femenina "insumisa" en dos sonetos del Trecento" publicado en Las revolucionarias. Literatura e insumisión femenina ed. de E. González de Sande y A. Cruzado Rodríguez, Sevilla, Arcibel, 2009, pp. 221-229

2 Cfr. S. Battaglia, Grande Dizionario della lingua italiana, UTET, Torino, 1960, (4) compiuto. Entre los poetas sículo-toscanos, Neri de' Visdomini en la canción Lo mio gioioso core se dirige a la dama "Gioia siete di Toscana/de le donne reina,/compiuta e fina di tutta valenza" (28.3; vv. 14-16). Cfr Poeti siculo-toscani (ed. R. Coluccia) en I Poeti della Scuola Siciliana (dir. C. Di Girolamo), Centro di Studi filologici e linguistici siciliani, Milano, Mondadori, 2008, vol. III. (citaremos en el cuerpo del texto con la abreviatura: PST).

3G. Contini considera las composiciones anónimas sin embargo, Stefano Carrai las atribuye a Maestro Rinuccino. Vid. I sonetti di Maestro Rinuccino da Firenze ed. de S. Carrai, Firenze, Accademia della Crusca, 1981, p. 118-124. Seguiremos para el corpus de la Compiuta Donzella la edición de G. Contini, Poeti del Duecento, Milano, Ricciardi, 1960, (2 t.). Utilizaremos la abreviatura PDI y PDII, para cada uno de los dos tomos respectivamente.

4 G. Contini incluye la composición en el corpus de Guido Guinizzelli pero indica que otros testimonios lo adjudican a Maestro Rinuccino. Vid. G. Contini, (a cura di) Poeti del Duecento, Milano-Napoli, Ricciardi, 1960, t. II, p. 474. S. Carrai lo atribuye claramente a Maestro Rinuccino. Vid. op. cit., pp. 123-124. e cusì più ch'altra donna terrena dovete intendere a Lui servire e amare de tutto corale amore e de pura e de compita fede." (Margueron 1990: 88). Todas estas referencias han llevado a la crítica, en un principio, a entablar un debate y centrar su atención en la cuestión de la autoría debido, como decíamos, a la ambigüedad del nombre y a la falta de pruebas documentales que avalen la existencia de una mujer trovadora. Algunos estudiosos se han apoyado en argumentos tan débiles, para demostrar su realidad, como la feminidad de los textos o, al menos, consideran su poesía como idealmente característica de una mujer real (Bertoni 1910: 101), (Chiari 1954: 5). Otros autores declaran improbable su existencia, dado el contexto histórico italiano medieval, muy distinto al de la Provenza, además de aducir como argumento la falta de referencias históricas explícitas a esta autora (Borgognoni 1891: 163). Otros estudios, sin afirmar abiertamente una autoría femenina, presuponen que detrás de la rúbrica hay una mujer, cuando critican la técnica rudimentaria de la composición y ponen en entredicho su formación comparándola a la de una "scolaretta in età puberale" (Crespo 1988: 222). Más recientemente, los estudios han focalizado su interés en el análisis de tipo textual, que demuestra la estrecha relación entre los dos sonetos atribuidos a la Compiuta Donzella hasta el punto de ser interpretados como una dúplice composición que desarrolla en el primer soneto el tópico primaveral que invita al amor, al tiempo que la protagonista sufre dolor y penas por el deseo del padre de casarla a la fuerza, mientras que el segundo soneto manifiesta su auténtico deseo de servir a Dios, enmarcándolos en el contexto guittoniano de rechazo al amor cortés y mundano a favor del amor espiritual (Carrai 1992: 207-213). Steinberg pone el énfasis en la función de estas composiciones dentro del códice, apuntando a una intención paródica o jocosa por parte de los compiladores del manuscrito, que han incluido los textos estratégicamente, junto con otros poemas pertenecientes al código de la canción de mujer, al final de cada uno de los cuadernos "come risposta e come critica alla tradizione cortese costituita dalle poesie che inmediatamente le precedono" (Steinberg 2006: 15). El estudio plantea la interesante hipótesis de un orden dialógico y narrativo entre los poemas pertenecientes a la lírica "alta" y las composiciones escritas en voz femenina que cuestionan la autenticidad del código cortés expresado, en los textos de los primeros autores del vulgar italiano, que ocupan el espacio más amplio del manuscrito.

Dejando a un lado la estéril cuestión de si la rúbrica se corresponde con una dama real o es el pseudónimo de un autor, nos parece más interesante centrar nuestro trabajo en el análisis interno de los textos puestos en boca de una voz femenina y abordar el estudio de las composiciones enmarcándolas en el contexto románico, en relación con otras tradiciones medievales en las que la focalización narrativa corresponde a una voz femenina. No incluiremos en este estudio el soneto en tenzone, que desarrolla el tema 
del servicio amoroso dentro del código cortés y que se aleja, por tanto, del motivo que sirve de inspiración a las otras dos composiciones.

\section{Corpus}

Las tres composiciones que le son atribuidas son tres: A la stagion che 'l mondo foglia e fiora, Lasciar vorria lo mondo e Dio servire y Ornato di gran pregio e di valenza, esta última dentro de una tenzone (PDI, p. 434-437). El primero de los sonetos A la stagion che 'I mondo foglia e fiora desarrolla en el exordio el tópico del locus amoenus y el cliché literario del buen tiempo y la estación primaveral, utilizado como contrapunto a su estado de ánimo ya que mientras los amantes corteses cumplen con el servicio amoroso a ella le embarga la tristeza y el sufrimiento ante la pretensión del padre de casarla contra su voluntad y no porque ponga reparos a un pretendiente concreto sino porque, simplemente, ante el cambio de estado: "non ho disio né voglia":

$$
\begin{aligned}
& \text { A la stagion che ' } 1 \text { mondo foglia e fiora } \\
& \text { acresce gioia a tutti fin' amanti: } \\
& \text { vanno insieme a li giardini alora } \\
& \text { che gli auscelletti fanno dolze canti; } \\
& \text { la franca gente tutta s'inamora, } \\
& \text { e di servir ciascun tragges' inanti, } \\
& \text { ed ogni damigella in gioia dimora; } \\
& \text { e me, n'abondan marrimenti e pianti. } \\
& \text { Ca lo mio padre m'ha messa 'n errore, } \\
& \text { e tenemi sovente in forte doglia: } \\
& \text { donar mi vole a mia forza segnore, } \\
& \text { ed io di ciò non ho disio né voglia, } \\
& \text { e 'n gran tormento vivo a tutte l'ore; } \\
& \text { però non mi ralegra fior né foglia. } \\
& \text { (PDI, I, 434) }
\end{aligned}
$$

La composición, desde el punto de vista de la focalización narrativa, la voz femenina perteneciente a una joven doncella que se opone a los deseos paternos, podría ponerse en relación con otras composiciones que forman parte del corpus lírico medieval de la canción de mujer, del que tenemos ejemplos en todas las tradiciones romances. Sin embargo, el tema del rechazo al matrimonio llama la atención puesto que en este código lo habitual es que, en las composiciones que tienen como protagonista a la muchacha soltera, el tema sea el del amor prohibido por parte de la familia, o por el contrario, el de la muchacha bramosa di marito. Suelen ser baladas que se presentan en forma de diálogo con la madre o el padre (PDI, p. 784). El segundo soneto, relacionado con el anterior a través de una serie de paralelismos lingüísticos y temáticos y de un mismo esquema métrico, ha de ser leído como continuación de la primera composición (Carrai 1992: 209-210):

Lasciar vorria lo mondo e Dio servire

e dipartirmi d'ogne vanitate,

5 Seguimos la edición de G. Contini, (t. I) utilizando la abreviatura PDI. pero che veggio crescere e salire

mattezza e villania e falsitate,

ed ancor senno e cortesia morire

e lo fin pregio e tutta la bontate:

ond'io marito non vorria né sire,

né stare al mondo, per mia sire,

né stare al mondo, per mia volontate.

di ciaschedun son forte disdegnosa,

di ciaschedun son forte disdegnosa,
e verso Dio la mia persona torna.

e verso Dio la mia persona torna.
Lo padre mio mi fa stare pensosa,

Lo padre mio mi fa stare pensosa,
ca di servire a Cristo mi distorna:

non saccio a cui mi vol dar per isposa.

(PDI, II, 435).

El motivo desarrollado, que expone el argumento último del sufrimiento que manifiesta en la primera composición, tampoco se ajusta a las convenciones conceptuales de la canción de mujer, puesto que en este registro poético lo habitual es que la temática gire alrededor de tres ejes: el amor correspondido, el amor no correspondido y el amor prohibido. En esta composición la joven, ante los deseos del padre de casarla, muestra su rechazo al sexo masculino: c'ogn'om di mal s'adorna,/di ciaschedun son forte disdegnosa (II, 9-10), su desacuerdo con respecto al contrato matrimonial: ond'io marito non vorria né sire (II, 7) y su deseo de servir a Cristo, firme propósito que se plantea ya en el incipit y que volvemos a encontrar en el penúltimo verso del soneto, con la contraposición de la forma verbal vorria del inicio y el distorna que hace referencia a la oposición del progenitor: Lo padre mio mi fa stare pensosa,/ca di servire a Cristo mi distorna (II, 12-13).

\section{Contexto Románico}

No hemos encontrado en el corpus de la canción de mujer en la lírica románica ningún texto que desarrolle los argumentos que hemos señalado. Tampoco por lo que se refiere a la homogeneidad formal estos textos coinciden con los rasgos característicos del código de la canción de mujer, puesto que la mayoría introducen procedimientos que se consideran "populares" si bien es cierto que en este código no faltan textos pertenecientes a la llamada "lírica alta". Aunque no faltan textos que forman parte del nivel áulico, y que pertenecen a los que podría determinarse "hegemonía del arte", otros introducen procedimientos que se consideran [...] "populares" como el paralelismo, la asonancia, el leixa-pren" entre otros, que obedecen al carácter eminentemente tradicional del código y que parecen indicar que la estructura tenía, al menos entre los siglos XII y XIV, una jerarquía y una funcionalidad diferentes con respecto a la estética cortés" (Lorenzo 1990: 271).

Corrado Bologna, haciéndose eco de las dudas sobre la existencia de la autora, la menciona como un epígono tardío de las trovairtz provenzales (Bologna 1995: 450) 
y es que, en efecto, los rasgos señalados anteriormente vinculan de alguna manera los sonetos con esta tradición aunque, paradójicamente, esta perspectiva presupone admitir una autoría femenina, hipótesis, que como hemos expuesto al principio, no cuenta con los datos suficientes para ser sostenida con rotundidad. Pero veamos de que manera podemos relacionar estos sonetos con los textos compuestos por las trovadoras provenzales.

En la sociedad trovadoresca del sur de Francia entre los siglos XII y XIII, en la que florecen la cultura y la literatura corteses, un grupo de mujeres pertenecientes en su mayoría, a la aristocracia fueron no sólo objeto de representación en los poemas del gran chant courtois sino también objeto de escritura literaria. No son muchas las composiciones conservadas y faltan datos y noticias que ayuden a completar la biografía de muchas de ellas. La información con la que contamos es, en bastantes casos, indirecta, a través de menciones realizadas por sus coetáneos, por eruditos renacentistas o por las composiciones de los trovadores con los que mantuvieron correspondencia (Martinengo 1977: 44). Sin embargo, de algunas, conocemos directamente su biografía como es el caso de Azalais de Porcairagues, Castelloza, la condesa de Día, Lombarda y Tibors; de otras sabemos, al menos, los motivos que las llevaron a componer como Almucs, Clara de Anduza y Maria de Ventadorn, gracias a las vidas y razós que se encuentran intercaladas entre las composiciones recogidas en los cancioneros. (Liborio 1982: 144-152). El lenguaje de estas mujeres y las situaciones que plantean son diametralmente opuestos a los de sus colegas masculinos. Se prefiere un estilo directo, un discurso más conversacional y no se idealizan las relaciones sobre las que escriben. Entre los temas que tratan: el lamento por la frialdad de un enamorado, la intercesión a favor de un amante que ha cometido un error, cuestiones de ética amorosa o asuntos de tema político, un grupo de composiciones expresan la petición de consejo, bien entre mujeres o bien hombres que acuden a la sabiduría femenina para resolver un entuerto amoroso. Dentro de este último grupo, contamos con una tensón en la que dos jóvenes piden consejo sobre su futuro a una mujer más experta o de mayor experiencia:

$\mathrm{Na}$ Carenza al bèl còrs avenenz,

donatz conselh a nos doas serors,

e car saubetz mielhz triar la melhors,

conseilhatz mi second vòstr'escienz:

pen $r$ ai marit a vòstra conoissença,

o'starai mi pulcela? E si m'agença,

que far filhons non cuit que sia bos

e sens marit mi par tròp angoissós.

N'Alaisina Yselda, 'nsenhamenz

prètz e beltatz, jovenz, frescas colors

conosc qu'avetz, cortisia e valors,

sobre totas las autras conoissen

per qu'ie us conselh per far bona semença penre marit coronat de sciença,

en cui faretz fruit de filh gloriós

Retenguda's pulcel' a cui l'espós.

$\mathrm{Na}$ Carenza, penre marit m'agença

mas far infanz cuit qu'es gran penitença,

que las tetinas pendon aval jos

e lo ventrilh es rüat e'nojós.

N'Alaisina Yselda sovinença

Ajatz de mi in l'umbra de guirença:

Ajatz de mi in l'umbra de guirença:

Qu'al departir mi ritenga près vos.

(Rieger, 1991: 155).

No hay noticias sobre ninguna de las tres protagonistas de esta composición. Podemos deducir de la lectura del texto que dos damas jóvenes y pertenecientes a un ambiente noble y refinado, Alais e Iselda, (prètz e beltatz, jovenz, frescas colors/ conosc qu'avetz, cortisia e valors vv.10-11) se dirigen a Carenza, otra dama de mayor experiencia y sabiduría para consultarle sobre la conveniencia de tomar marido o permanecer vírgenes, porque si bien la presencia de un cónyuge no se rechaza abiertamente, la maternidad es vista como un lastre que sólo acarrea trabajos e inconvenientes, puesto que supone la deformidad del cuerpo y la pérdida de la lozanía. Carenza les propone tomar como marido a coronat de scienza, que ha sido interpretado como una referencia a Cristo y la recomendación de una consagración a la vida espiritual (Rieger 1991: 157)

Esta alternancia de interlocutores viene también marcada por un lenguaje en el que las frases elevadas y refinadas de la dama se codean con el registro más vivo y coloquial de las jóvenes doncellas. Podríamos decir que se trata de una creación ecléctica en la que se combinan tonos populares (que las tetinas pendon aval jos e lo ventrilh es rüat énojós (vv. 19-20) con elementos de la canción amorosa ${ }^{6}$.

\section{Conclusión}

La crítica al matrimonio y el rechazo de la maternidad, junto con la presentación de la alternativa de la vida monástica y la consagración a Cristo, ponen de alguna manera en relación la tradición textual de las composiciones de las trovairitz con las composiciones atribuidas a la Compiuta Donzella, sean o no fruto del ejercicio literario de una mujer, ya que creemos que gravitan en la órbita de un mundo cortés en el que se reflejan, aún formando parte de un juego artificioso y convencional, otras sensibilidades

6 Esta intención irónica y la visión crítica del matrimonio o, al menos, de la maternidad, es algo que también ha sido destacado por la crítica y alguna autora, ha aventurado la conexión del texto con el fenómeno del catarismo, movimiento religioso, surgido en la Francia meridional y declarado herético por la ortodoxia cristiana hasta el punto de que el Papa Inocencio III va a promover la cruzada contra los albigenses a partir del año 1209. Esta doctrina que en su interpretación más extrema recomendaba la abstención de relaciones sexuales y mostraba un rechazo radical frente al matrimonio asignaba a las mujeres un importante papel ya que compartían con los hombres la función sacerdotal e incluso predicaban ante el público femenino. Vid. M. Martinengo, op. cit., p. 32. 
y aspiraciones distintas a las funciones que tradicionalmente se le asignaban a las mujeres. Steinberg cree que el debate que pretenden los compiladores del Vaticano 3793 y la función paródica que tal vez se esconda tras la atribución de los textos a una mujer quizás responda: "al disagio dei lettori e degli scrittori coevi, provocato dalle differenze fra una tradizione letteraria che assegna alla donna un ruolo di autrice e protagonista e le effettive condizioni storiche della società mercantile fiorentina, fra l'appropiazione di una poesia "in voce di donna" e la mancanza di un suo riferimento extratestuale." Vid. (2006: 15).

\section{REFERENCIAS BIBLIOGRÁFICAS}

Battaglia, S., Grande Dizionario della lingua italiana, Torino, UTET, 1960.

Bogin, M., Les Trovairitz, Barcelona, laSal, 1983.

Bologna, C., "Poesia del centro e del nord" en Storia della letteratura italiana (dir. E. Malato), vol. I Dalle origini a Dante, Roma, Salerno, 1995, pp. 405-525.

Carrai, S. (a cura di), I sonetti di Maestro Rinuccino da Firenze, Firenze, Accademia della Crusca, 1981.

----, “Il dittico della Compiuta Donzella”, Medioevo Romanzo, XVII (1992), pp. 207-213.

Coluccia, R., (a cura di) Poeti siculo-toscani en I poeti della Scuola siciliana, (dir. C. Di Girolamo), Edizione promossa dal Centro di studi filologici e linguistici siciliani, Milano, Mondadori, 2008, vol. III.

Crespo, R., “La Compiuta Donzella”, Medioevo Romanzo, XIII (1988), pp. 203-233.

Contini, G., Poeti del Duecento, Milano-Napoli, Ricciardi, 1960, (2 vols.).

Liborio, Maㅗ A., Storie di dame e trovatori di Provenza, Milano, Bompiani, 1982.

Lorenzo, P., La canción de mujer en la lírica medieval, Santiago de Compostela, Servicio de Publicaciones e Intercambio científico, 1990.

Lubello, S., (a cura di) "Maestro Torrigiano" in I Poeti della Scuola Siciliana, Milano, Mondadori "I Meridiani", 2008, vol. 3.

Margueron, C., (a cura di) Guittone D'Arezzo. Lettere, Bologna, Commissione per i testi di lingua, 1990.

Martinengo, M., Las trovadoras poetisas del amor cortés, (trad. Rivera Garretas, Mª M.,-Martín Domínguez, S.), Madrid, Grafistaff, 1997.

Rieger, A., Trobairitz, Tübingen, Max Niemeyer, 1991.

Riquer, M. de, Los trovadores. Historia literaria y textos, Barcelona, Planeta, 1975, 3 vols.

Steinberg, J., "La Compiuta Donzella e la voce femminile nel manoscritto" Giornale storico della letteratura italiana, CLXXXIII, fasc. 601 (2006), pp. 1-31. 